\title{
DISCUSIONES SOBRE LA PRESENCIA DE AVES \\ RAPACES, AVES MIGRATORIAS Y AVES BAJO ALGÚN \\ GRADO DE AMENAZA EN LA CIUDAD DE PEREIRA, RISARALDA
}

\author{
JUAN CARLOS LONDOÑO-BETANCOURTH ${ }^{1}$
}

Recibido el 27 de agosto de 2012 y aprobado el 6 de noviembre de 2012

\section{RESUMEN}

Artículo que presenta una discusión que busca justificar la presencia de aves silvestres dentro de la ciudad de Pereira, enfáticamente las especies de aves rapaces, migratorias o bajo algún grado de amenaza, buscando ampliar la discusión de resultados presentada en el artículo predecesor llamado "Revisión a la diversidad ornitológica de Pereira". Se registraron catorce(14) especies de aves rapaces (es decir pertenecientes a las familias Cathartidae, Pandionidae, Accipitridae y Falconidae) las cuales deben su presencia a que no dependen de bosque y pueden sobrevivir en zonas urbanas; veintiséis (26) especies migratorias de las cuales, a excepción de tres especies consideradas raras, todas son comunes o bastantes comunes y la mayoría frecuenta áreas abiertas; siete (7)especies bajo algún grado de amenaza(amenaza que se debe principalmente a la destrucción de su hábitat) consideradas de por sí como raras y locales y cuatro (4) especies endémicas de las cuales solo una es considerada como rara, todas las demás son comunes y dentro de la ciudad encuentran los diversos tipos de hábitat requeridos por estas especies. Se discute y concluye que en general las especies abordadas son de áreas abiertas o no dependen de bosques, tolerantes a ecosistemas intervenidos y/o presentan cierta plasticidad de nicho con sus necesidades de hábitat y son capaces de encontrar sus requerimientos ecológicos dentro de las zonas urbanas. Así mismo que la heterogeneidad de hábitats y la estructura de vegetación variada de las zonas urbanas contribuyen a su riqueza de especies.

\section{PALABRAS CLAVE}

Aves, rapaces, migratorias, en peligro, Pereira.

\section{DISCUSSIONS ABOUT THE PRESENCE OF BIRDS OF PREY, MIGRATORY BIRDS AND BIRDS UNDER SOME DEGREE OF THREAT IN THE CITY OF PEREIRA, RISARALDA}

\begin{abstract}
The article presents a discussion that seeks to justify the presence of wild birds in the city of Pereira -emphatically birds of prey, migratory birds, or birds under some degree of threatseeking to broaden the discussion of results presented in the
\end{abstract}


previous article "Revision to Ornithological Diversity in Pereira." Fourteen (14) species of birds of prey (this is to say belonging to the families Cathartidae, Pandionidae, Accipitridae and Falconidae) which owe their presence to the fact that they do not depend on forests and can survive in urban areas; twentysix (26) migratory species which, except for three species considered rare, all are common or fairly common and most frequent in open areas; seven (7) species under some degree of threat (threat mainly due to habitat destruction) considered as rare and local; and four (4) endemic species of which only one is considered rare; all others are common and within the city they find the different types of habitat they require. It is discussed and concluded that in general the species dealt with belong to open areas and do not depend on forests, are tolerant to intervened ecosystems and/or have some niche plasticity with their habitat needs and are able to find their ecological requirements in urban areas. Also that the habitat heterogeneity and the varied vegetation structure in urban areas contribute to the richness of species.

\section{KEYWORDS}

Wild birds,birds of prey, migratory birds, endangered birds, Pereira.

\section{INTRODUCCIÓN}

Los asentamientos humanos como las áreas urbanas en Colombia se encuentran sobre ecosistemas biodiversos, ejemplo de ello son:

La ciudad de Medellín sobre el Valle de Aburrá, un gran ecosistema en el que se registran más de 400 especies de aves distintas (SAO, 2003); o la ciudad de Bogotá, que se desarrolló sobre la Sabana de Bogotá, un ambiente en el que se registran cerca de 200 especies de aves (ABO, 2000), aunque está altamente urbanizada. [...] La gran diversidad de aves de Colombia no sólo se refleja en sus ecosistemas, como en el Valle de Aburrá o la Sabana de Bogotá, sino que se evidencia en los parques y jardines de los municipios y ciudades de todo el país (Osorio \& Molina, 2009, pp. 50-51)

La capacidad de vuelo y la adaptabilidad, ha permitido a algunas especies de aves no restringir su hábitat a ecosistemas nativos como bosques primarios o lagos de aguas límpidas.

[...] muchas especies se han adaptado a ambientes totalmente artificiales o alterados por los humanos, como las áreas urbanas y rurales. [...] Otras especies no son tan exigentes, de 
manera que habitan o frecuentan las áreas verdes y en los árboles urbanos de la ciudad, donde encuentran alimento y un lugar para construir el nido (Osorio \& Molina, 2009, p. 51)

"La urbanización es un proceso continuo que produce una gama de diferentes densidades y patrones de asentamiento humano" (Marzluff et al.,2001 citados en Villegas\& Garitano, 2008, p. 146), "provocando la reducción y fragmentación de la vegetación nativa y modificando las comunidades de fauna residentes" (Marzluff \& Ewing, 2001 y Alberti et al.,2003 citados en Villegas \& Garitano, 2008, p. 146).Las aves son indicadores biológicos muy eficientes que, con su presencia o ausencia, indican la calidad ambiental en áreas naturales, rurales o urbanas. Un ave que deja de observarse en un parque o en una ciudad, indica el deterioro ambiental del parque o de la ciudad. "Por su diversidad y movilidad las aves pueden decirnos mucho acerca de los cambios ambientales; la disminución de las especies o sus poblaciones, indica deterioros en el ambiente" (BirdLife, 2002, p. 7 citado en Osorio \& Molina, 2009, p. 52). "El uso de especies de aves como indicadoras se realiza bajo el supuesto de que las respuestas de especies individuales pueden ser representativas de la respuesta a otra fauna en la comunidad" (Macnally \& Fleishman, 2004 y Fleishman et al.,2005 citados en Villegas \& Garitano, 2008, p.146).

En el presente estudio se toma a las aves como un indicador ecológico de los ecosistemas urbanos, donde la presencia de ciertas especies señala que existen hábitats que pueden satisfacer sus necesidades de alimento y refugio temporal o permanentemente, "tomando en cuenta que cada una puede responder independientemente a la variación ambiental" (Canterbury et al.,2000 citado en Villegas \& Garitano,2008, p. 146) "y que la presencia o ausencia de una de éstas, puede indicar condiciones ecológicas particulares" (Carignan \&Villard, 2002 citados en Villegas \& Garitano, 2008, p. 146).

En esta investigación se pueden discriminar distintas especies de aves. Así, estudios de corta duración con criterios relativamente simples pueden mostrar características de la avifauna en relación a disturbios ambientales en un ecosistema urbano, de forma rápida y a bajo costo que pueden contribuir a identificar las acciones necesarias para contribuir a la recuperación y conservación de los ecosistemas que sostienen las poblaciones de estas especies. "En un proceso cuya finalidad sea seleccionar especies indicadoras, el primer paso es determinar los objetivos de la indicación y luego indicadores potenciales pueden ser seleccionados basándose en un grupo de criterios a priori" (Niemelä, 2000 citado en Villegas \& Garitano,2008, p. 149). "Posteriormente debe establecerse la relación entre el indicador y el estado ecológico o ambiental" (Lindenmayer, 1999 y Niemelä, 2000 citados en Villegas \& Garitano, 2008, p.149). "Si la correlación existe, se debe establecer la solidez del indicador mediante el planteamiento y evaluación de diferentes hipótesis" (Niemelä, 2000 citado en Villegas \& Garitano, 2008, p.149).En este estudio únicamente se dio el primer paso. Para seguir todo el proceso e identificar a 
las especies de aves indicadoras de características particulares falta aún medir y establecer la relación entre estas y los factores ambientales de urbanización. Este sería un importante aporte en el futuro.

El uso de aves como indicadores también tiene desventajas, porque "las aves no necesariamente pueden reflejar la salud de otros taxones que viven en el mismo hábitat" (Gregory, 2006 citado en Villegas \& Garitano,2008, p. 149). Además, "las aves pueden tener respuestas diferenciales a los disturbios en relación a otros grupos de organismos" (Lindenmayer,1999 y Milesiet al.,2002 citados en Villegas \& Garitano, 2008, p.149) y no se pueden hacer generalizaciones para toda la comunidad biológica. Por otro lado, la presencia actual de una especie puede no ser un buen indicador de su futura persistencia, ya que "las extinciones pueden ocurrir después de periodos prolongados de un disturbio" (Lindenmayer,1999 citado en Villegas \& Garitano, 2008, p.151).

Al evaluar la presencia de especies de aves rapaces, migratorias o bajo algún grado de amenaza dentro de los ecosistemas urbanos y los elementos ecológicos que lo permiten, se adquieren criterios para la toma de decisiones en aras de conservar los hábitats de estas especies(sean reductos naturales o espacios antrópicos) que sustenten las poblaciones de estas y otras especies asociadas dentro de las comunidades de aves, así como evaluar el grado de impacto de la urbanización en la población de alguna especie determinada y su estado de conservación.

\section{METODOLOGÍA}

\section{Área de estudio}

El municipio de Pereira está localizado a 4 grados 49 minutos de latitud norte, 75 grados 42 minutos de longitud y 1411 metros sobre el nivel del mar; en el centro de la región occidental del territorio colombiano, en un pequeño valle formado por la terminación de un contrafuerte que se desprende de la cordillera Central (Figura 1). 


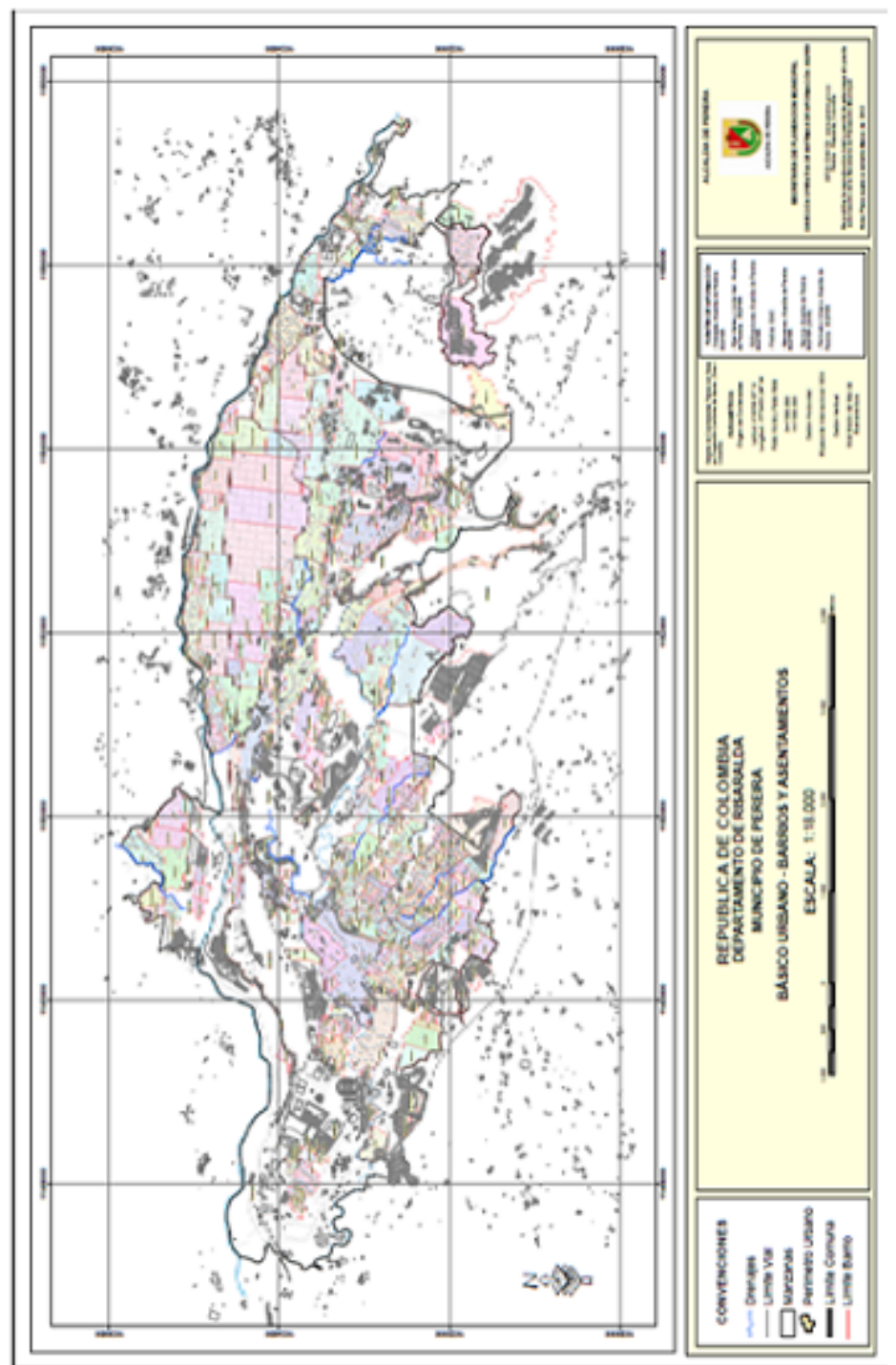

Figura 1. Mapa de localización de área de estudio. Fuente: Alcaldia de Pereira, 2009.

La ciudad de Pereira cuenta con una temperatura promedio de $21^{\circ} \mathrm{C}$, y su precipitación media anual es de $2750 \mathrm{~mm}$.

\section{Colección de datos}

El presente documento es una ampliación de la discusión de resultados del artículo "Revisión a la diversidad ornitológica de Pereira" del mismo autor (Londoño, 2011), el cual se elaboró con fuentes de información primarias (observación en campo) y secundarias (consulta bibliográfica). 


\section{RESULTADOS Y DISCUSIÓN}

Dentro de los estudios de biodiversidad urbana y en este caso ornitológica, existen elementos de peso que obligan a considerar que estas poblaciones son pobres en número de especies e individuos por especie, dado que el establecimiento de asentamientos humanos como las urbes:

A menudo resultan en la creación de un paisaje en el cual quedan algunos parches de vegetación natural, impresos en una matriz de hábitats modificados por actividades humanas. Esta fragmentación de hábitats naturales constituye una amenaza para la preservación de la biodiversidad. La fragmentación causa cambios en el ambiente físico a nivel regional (e. g. humedad, temperatura) y puede llevar a la extinción de altas proporciones de la flora y fauna locales (Bierregaard \& Lovejoy, 1989; KATTAN et al., 1994; Lovejoy et al., 1986 y Saunders et al., 1991 citados en Serrano \& Aparicio, 1996)

Las extinciones causadas por fragmentación resultan principalmente a consecuencia de dos fenómenos. Primero, la fragmentación simplifica drásticamente el paisaje, pues lo que antes era un paisaje florísticamente denso y fisionómicamente complejo y heterogéneo, se convierte en unos parches aislados en una matriz de hábitat altamente homogenizado y adverso para la vida silvestre (Lovejoy et al., 1986; Saunders et al., 1991). Y segundo, la fragmentación reduce el área total de bosques y aísla pequeñas poblaciones en los parches remanentes (Newmark, 1991; Willis, 1979). La reducción en el área causa la extinción de especies que requieren grandes áreas de actividad. Por otra parte, las poblaciones de especies que quedan aisladas en los fragmentos pueden sufrir altas tasas de extinción (Serrano \& Aparicio, 1996)

Pero los ecosistemas urbanos aunque no tan ricos en diversidad paisajística y de hábitat y a pesar de ser considerados pobres desde una perspectiva ecológica en elementos que contribuyan a la conservación sustentable de poblaciones de fauna silvestre nativa, poseen una variada diversidad espacial representada en parques, jardines, lotes baldíos, edificaciones, zonas rivereñas, campos abiertos, vegetaciones multiestrato en diversos estados sucesionales, entre otras (Tabla 1) que contribuyen de algún modo y muy modesto a:

[...] la riqueza local y regional de comunidades de aves neotropicales (Levey \& Stiles, 1994). 
Esta heterogeneidad [...] incluye en la composición y riqueza de las comunidades de especies en los claros y el interior de la ciudad (Levey, 1988b; Schemske \& Brokow, 1981 y Wonderle et al., 1987) (Serrano \& Aparicio, 1996)

Tabla 1. Area de investigación: Trayectos y caracterización ecológica

\begin{tabular}{|c|c|}
\hline Trayecto & Caracterización Ecológica \\
\hline $\begin{array}{l}\text { 1- Esperanza -Galida, } \\
\text { Parque Recreacional } \\
\text { Comfamiliar Galicia }\end{array}$ & $\begin{array}{l}\text { Caracterizado por ecosistemas de sabana de áreas abiertas con pastizales, } \\
\text { árboles y arbustos dispersos, bosques secundarios con claros y en diversos } \\
\text { estados sucesionales, humedales y rios favoreciendo la presencia de } \\
\text { diversas especies de aves. }\end{array}$ \\
\hline $\begin{array}{l}\text { 2- Corregimiento Tribunas - } \\
\text { Córcega }\end{array}$ & \begin{tabular}{|l}
$\begin{array}{l}\text { Posee ecosistemas de bosque secundario en diversos estados } \\
\text { sucesionales (bosque de niebla). }\end{array}$ \\
\end{tabular} \\
\hline $\begin{array}{l}\text { 3- Campus y Jardin Botánico } \\
\text { Universidad Tecnológica de } \\
\text { Pereira }\end{array}$ & $\begin{array}{l}\text { Es una zona con abundantes relictos boscosos poco intervenidos por } \\
\text { actividades antrópicas o que han sido abandonados permitiendo la } \\
\text { recuperación de la cobertura vegetal en multiestratos; el campus de la } \\
\text { Universidad Tecnológica y su Jardín Botánico se caracterizan por poseer } \\
\text { ecosistemas de bosque de guadua, bosque secundario en diferentes } \\
\text { estados sucesionales (bosque andino) yun humedal. }\end{array}$ \\
\hline $\begin{array}{l}\text { 4- Quebrada El Oso tramo } \\
\text { Cuba, rio Consota tramo } \\
\text { barrios Los Corales y } \\
\text { Gamma (comuna Olímpica), } \\
\text { parque central de Cuba, } \\
\text { barrio Belmonte y Central } \\
\text { Mayorista de Alimentos, } \\
\text { MERCASA } \\
\text { Olimpica) } \\
\end{array}$ & $\begin{array}{l}\text { Hace parte del área urbana de la ciudad, es una zona cruzada por el río } \\
\text { Consota y la quebrada El Oso, abundan los rodales de guadua, zonas } \\
\text { abiertas y semiabiertas algunas con árboles dispersos y/o arbustos en } \\
\text { diversos estados sucesionales, pastizales, jardines y parques, rodales de } \\
\text { caña brava, bosques de galeria y zonas habitacionales. }\end{array}$ \\
\hline $\begin{array}{l}5 \text { Barrio El Plumón, vía de } \\
\text { accesorapuerto } \\
\text { Internacional Matecaña y } \\
\text { Zoológico Matecaña }\end{array}$ & $\begin{array}{l}\text { También hace parte del área urbana, se caracteriza por poseer zonas } \\
\text { abiertas y pastizales, bosque seaundario (bosque andino) y jardines asi } \\
\text { comoáreas habitacionales. }\end{array}$ \\
\hline $\begin{array}{l}\text { 6- barrios Perla del sur, } \\
\text { Laureles II, Comfamiliar, El } \\
\text { Edén, El Cardal, Tinajas, } \\
\text { Gibraltar y Portales de } \\
\text { Birmania (comuna San } \\
\text { Joaquin) }\end{array}$ & $\begin{array}{l}\text { Es un área mayoritariamente urbanizada con áreas semiabiertas, zonas } \\
\text { con arbustos y árboles, rodales de guadua, jardines y cultivos de café y } \\
\text { pequeños cultivos de plátano. }\end{array}$ \\
\hline $\begin{array}{l}\text { 7- Quebrada La Dulcera, } \\
\text { puente de acceso a } \\
\text { Universidad Católica Popular } \\
\text { de Risaralda, Batalón San } \\
\text { Mateo Avenida de las } \\
\text { Américas }\end{array}$ & $\begin{array}{l}\text { Es cruzado por el rio Consota, posee zonas abiertas y semiabiertas con } \\
\text { árboles dispersos y arbustos, bosque de galeria, rodales de guadua. }\end{array}$ \\
\hline 8-Comuna Centro & Constituye al centro de la ciudad, solo presenta jardines y parques. \\
\hline
\end{tabular}

Así, a pesar de ser los ecosistemas urbanos ambientes altamente perturbados por las actividades humanas donde poco o nada sobrevive de la diversidad ecosistémica y paisajística original, la diversidad espacial que ofrece "es un factor importante para los cambios en la estructura y composición de la vegetación por sucesión secundaria llevando asociados cambios en los ensamblajes de aves" (Loiselle \& Blake,1994 citados en Serrano \& Aparicio,1996). "De esta forma, las ricas comunidades son el resultado de la combinación en ensamblajes diferentes asociados con patrones de heterogeneidad espacial y procesos ecológicos que operan a distintas escalas" (Karr \& Freemark, 1983; Karr et al., 1990; Kikkawa, 1982; Levey \& Stiles, 1994; Loiselle \& Blake, 1994; Martin \&Karr, 1986 y Thiollay 1988, 1994 citados en Serrano \& Aparicio, 1996).

La heterogeneidad de hábitats internos de cada parque y el efecto negativo que tiene el predominio del uso residencial de las áreas 
circundantes son factores determinantes de la riqueza de aves. Cuando esta heterogeneidad aumenta por presencia de cuerpos de agua (canales y reservorios- fuentes, charcas, bebederos), estructura de vegetación variada forestación, céspedes húmedos, comunidades de hierbas altas, vegetación pantanosa- así como la presencia de superficies pavimentadas, muros y suelo desnudo, también se incrementa la riqueza de especies (Faggi \& Perepelizin, 2006, p. 295)

La presencia de las aves también se debe a la presencia de árboles.

Las aves al igual que los humanos tienen una dieta predilecta, de manera que no encuentran su alimento en "cualquier" árbol, sino en aquellos que producen las semillas, las frutas, o el néctar que las alimentan; o en torno a los que atraen a los insectos que hacen parte de su dieta (Osorio \& Molina, 2009, p. 53)

Algunos de los árboles que hacen parte de la arborización de los espacios urbanos de uso público y privado de Pereira, y que colaboran con la alimentación y el hospedaje de las aves en Pereira, se presentan a continuación:

- Quiebrabarrigo: Trichanthera gigantea (Nectarívoras),

- Corozo: Aiphanes aculeata (Frugívoras),

- Guayacán Amarillo: Tabebuia chrysantha (Semilleras y nectarívoras),

- Guayacán Rosado: Tabebuia rosea (Semilleras y nectarívoras),

- Totumo: Crescentia cujete (Nectarívoras),

- Achiote: Bixa orellana (Insectívoras),

- Yarumo: Cecropia peltata (Insectívoras y frugívoras),

- Chagualo: Clusia multiflora (Frugívoras),

- Casco de Vaca: Bauhinia picta (Nectarívoras),

- Ariza o Árbol de la Cruz: Brownea ariza (Nectarívoras),

- Carbonero: Calliandra pittieri (Insectívoras)

- Guamo: Inga edulis (Insectívoras y nectarívoras),

- Aguacate: Persea americana (Insectívoras) (SAO, 2009, p. $26,48,56,62,64,68,86,90,116,118,126,130$ y 168)

Uno de los mayores problemas que deben enfrentar las aves en las ciudades es, que si bien encuentran árboles (aunque no muchos), por lo general son árboles ornamentales introducidos que no les ofrecen alimento. Esto se debe a que las arborizaciones urbanas de nuestras ciudades se basan en el uso intensivo de especies ornamentales introducidas (Osorio \& Molina, 2009, p. 53) 
Son así los elementos anteriormente expuestos los que pueden contribuir a justificar a la identificación preliminar de doscientas tres (203) especies de aves registradas en el área de estudio según Londoño(2011, p.86).Dentro de las cuales en este artículo se abordarán las aves rapaces, migratorias o bajo algún grado de amenaza, discutiendo sobre los elementos de juicio que justifican su presencia.

En el área de estudio se registraron catorce(14) especies de aves rapaces (es decir pertenecientes a las familias Cathartidae, Pandionidae, Accipitridae y Falconidae), veintiséis(26) especies migratorias y siete (7) especies bajo algún grado de amenaza según McMullan, Donegan \& Quevedo (2010) y Renjifo et al.(2002) (Tabla 2). 
Tabla 2. Aves rapaces, aves migratorias y aves bajo algún rango de amenaza presentes en la ciudad de Pereira

Listado de especies por orden de familias según: Remsen et al. (2009), Salaman et al. (2009) y McMullan, Donegan y Quevedo (2010),

\begin{tabular}{|c|c|c|c|}
\hline \multirow[b]{2}{*}{ Nombre cientifico } & \multirow[b]{2}{*}{ Nombre vulgar } & \multicolumn{2}{|c|}{$\begin{array}{r}\text { Estado } \\
\end{array}$} \\
\hline & & $\begin{array}{c}\text { Según McMullan, } \\
\text { Donegan y Quevedo } \\
(2010)\end{array}$ & $\begin{array}{l}\text { Según Renjifo et } \\
\text { al.(2002) }\end{array}$ \\
\hline \multicolumn{4}{|c|}{ CRACIDAE: (2) (Guacharacas, Pavas, Paujiles) } \\
\hline Penelope perspicax & Pava Caucana & EN & EN \\
\hline Aburria aburri & Pava Negra & NT & NT \\
\hline \multicolumn{4}{|c|}{ CATHARTIDAE: (2) (Gallinazos o Buitres Americanos) } \\
\hline Cathartes aura & Guala Cabecirroja & $\mathrm{Mb}$ & \\
\hline Coragyps atratus & Gallinazo Negro & & \\
\hline \multicolumn{4}{|c|}{ PANDIONIDAE: (1) (Aguila Pescadora) } \\
\hline Pandion haliaetus & Aguila Pescadora & $\mathrm{Mb}$ & \\
\hline \multicolumn{4}{|c|}{ ACCIPITRIDAE: (7) (Gavilanes, Aguilas y Aguilillas) } \\
\hline Elanus leucurus & Gavilán Maromero & & \\
\hline Ictinia plumbea & Aguililla Plomiza & & \\
\hline Accipiter striatus & Azor Cordillerano & & \\
\hline Accipiter bicolor & Azor Bicolor & & \\
\hline Buteo magnirostris & Gavilán Caminero & & \\
\hline Buteo platypterus & Gavilán Aliancho & $\mathrm{Mb}$ & \\
\hline Buteo albicaudatus & Gavilán Coliblanco & & \\
\hline \multicolumn{4}{|c|}{ FALCONIDAE: (4) (Halcones, Caracaras) } \\
\hline Caracara cheriway & Caracara Moñudo & & \\
\hline Mivago chimachima & Pigua & & \\
\hline Faico sparverius & Cernicalo Americano & & \\
\hline Faico peregrinus & Halcón Peregrino & Mb Ma & \\
\hline \multicolumn{4}{|c|}{ SCOLOPACIDAE: (1) (Andarríos, Becasinas) } \\
\hline Tringa solitaria & Andarríos Solitario & $\mathrm{Mb}$ & \\
\hline \multicolumn{4}{|c|}{ TROCHILIDAE: (1) (Colibríes) } \\
\hline Heliodoxa imperatrix & Diamante Emperador & C-end & \\
\hline \multicolumn{4}{|c|}{ THAMNOPHILIDAE: (1) (Hormigueros Arbóreos) } \\
\hline Thamnophilus muitistriatus & Batará Carcajada & C- end & \\
\hline \multicolumn{4}{|c|}{ TYRANNIDAE: (4) (Atrapamoscas) } \\
\hline Leptopogon rufipectus & Atrapamoscas Pechirrufo & C-end & \\
\hline Empidonax flaviventris & $\begin{array}{l}\text { Atrapamoscas } \\
\text { Buchiamarrillo } \\
\end{array}$ & acc Mb SA & \\
\hline Myiodynastes macuitatus & Sirirí Rayado & [Ma] & \\
\hline Tyrannus savana & Sirirí Tijeretón & [Ma] & \\
\hline \multicolumn{4}{|c|}{ VIREONIDAE: (1) (Verderones - Víreos) } \\
\hline Vireo olivaceus & Verderón Ojirrojo & [Mb Ma] & \\
\hline \multicolumn{4}{|c|}{ HIRUNDINIDAE: (2) (Golondrinas) } \\
\hline Pygochelidon cyanoleuca & Golondrina Blanquiazul & [Ma] & \\
\hline Hirundo rustica & Golondrina Tijereta & $\mathrm{Mb}$ & \\
\hline \multicolumn{4}{|c|}{ TURDIDAE: (2) (Mirlas y Solitarios) } \\
\hline Catharus fuscescens & Zorzal Rojizo & $\mathrm{Mb}$ & \\
\hline Catharus ustulatus & Zorzal Buchipecoso & $\mathrm{Mb}$ & \\
\hline \multicolumn{4}{|c|}{ THRAUPIDAE: (4) (Tángaras) } \\
\hline Ramphocelus flammigerus & Toche Enjalmado & C-end & \\
\hline Chlorochrysa nitidissima & Tángara Multicolor & VU & \\
\hline Dacnis Hartlaubi & Dacnis Turquesa & VU & VU \\
\hline Saltator cinctus & Saltador Chusquero & NT & VU \\
\hline \multicolumn{4}{|c|}{ CARDINALIDAE: (3) (Cardinales, Picogordos, etc,) } \\
\hline Piranga rubra & Piranga Abejera & $\mathrm{Mb}$ & \\
\hline Piranga olivacea & Piranga Alinegra & $\mathrm{Mb}$ & \\
\hline Pheucticus ludovicianus & Picogordo Degollado & $\mathrm{Mb}$ & \\
\hline
\end{tabular}




\begin{tabular}{|c|c|c|c|}
\hline \multicolumn{5}{|c|}{ PARULIDAE: (9) (Reinitas) } \\
\hline Vermivora chrysoptera & Reinita Alidorada & Mb NT & \\
\hline Leiothlypis peregrina & Reinita de Tennessee & Mb & \\
\hline Dendroica fusca & Reinita Gorginaranja & Mb & \\
\hline Setophaga ruticilia & Candelita Norteña & Mb & \\
\hline Mniotita varia & Cebrita Trepadora & Mb & \\
\hline Parkesia noveboracensis & Reinita Acuática & Mb & \\
\hline Parkesia motacilla & Reinita de Luisiana & Mb & \\
\hline Oporornis philadeiphia & Reinita Enlutada & Mb & \\
\hline Wilsonia canadensis & Reinita de Canadá & Mb & \\
\hline \multicolumn{4}{|c|}{ ICTERIDAE: (2) (Oropéndolas, Arrendajos, Turpiales) } \\
\hline Icterus galbula & Oriol de Baltimore & Mb & \\
\hline Hypopyrrhus \\
pyrohypogaster
\end{tabular}

Códigos. EN: En Peligro, VU: Vulnerable. NT: Cercano a Amenaza -Casi Amenazada, Mb: Migratorio boreal,

Ma: Migratorio austral, Acc: Accidental/Nagabundo, C-end: Casi-endémica. SA: Especies conocidas únicamente de San Andrés y Providencia. Fuente: Londoño (2011)

\section{CARACTERIZACIÓN DE LAS ÁREAS CON MAYOR POTENCIAL DE CONSERVACIÓN Y ESTUDIO DE LA HISTORIA NATURAL DE LAS AVES RAPACES, AVES MIGRATORIAS Y AVES BAJO ALGÚN GRADO DE AMENAZA DE PEREIRA (RISARALDA)}

Los trayectos número 1, 2 y 3 (Tabla 1) fueron identificados como los de mayor presencia de número de especies y por ende se convierten en puntos de interés para la conservación de sus ecosistemas nativos y de las aves silvestres objeto de estudio en esta investigación.

El campus y Jardín Botánico de la Universidad Tecnológica de Pereira es:

Un área destinada a la protección, investigación y conservación in-situ y ex-situ de las especies nativas del bosque andino, conformada por una zona de bosque secundario en proceso de regeneración natural en el que se han desarrollado procesos de enriquecimiento con nuevas especies, en este se estableció como objetivo principal mantener un conjunto de colecciones representativas de la flora andina de la región cafetera colombiana y se definió como prioridad el coleccionar especies de la zona que se encuentren en algún grado de vulnerabilidad y/o que sean de interés económico y/o cultural. De allí que se haya dado prioridad a la incorporación de especies como Cedro negro (Juglans neotropica), Chaquiro (Podocarpus oleifolium), Cedro de montaña (Cedrela montana), Comino crespo (Aniba perutilis), Sande (Brosimum utile), Cattleya quadricolor y Cattleya shoeredae, las cuales por uso, sobreexplotación y/o pérdida de hábitat se encuentran en peligro de extinción (Jardín Botánico Universidad Tecnológica de Pereira, s.f.) 
En los proceso de conservación del área del Jardín Botánico no solo se involucra la conservación de las especies vegetales propias de este ecosistema (bosque andino) sino también la de algunas especies animales asociados a ellas (creación de nichos o nuevas fuentes alimenticias).

\section{El corregimiento Tribunas -Córcega:}

Se localiza en una zona de gran potencial acuífero debido a la presencia de aguas subterráneas y manantiales, además cuenta con varias fuentes de agua superficiales como lo es el río Barbas, Consota y Cestillal y varias quebradas como lo son: Condina, Oso, Tinajas, Peñitas, Bizcochuelo, Viboral, La Mesa, San José, Balsora, entre otras. Algunas de estas fuentes superficiales además de ser fuentes abastecedoras de acueductos comunitarios, han llegado a ser identificadas como fuentes alternas de abastecimiento para la ciudad de Pereira (Caso del río Consota) (López \& Hernández, 2009, p.15)

La caracterización vegetal de río Consota es de "Bosques muy húmedos premontanos (bmh-PM) y Bosques muy húmedos montanos bajos (bmh-MB)" (López \& Hernández, 2009, p.58) y la del río Barbas es de "Bosques húmedos subtropicales" (López \& Hernández, 2009, p.60).

La precipitación en el corregimiento de Tribunas varía entre los $2200 \mathrm{~mm}$ y $3700 \mathrm{~mm}$ al año, con una precipitación media aproximada de $2900 \mathrm{~mm} / \mathrm{año}$ por lo cual se tiene una alta oferta de agua superficial y para recarga de acuíferos subterráneos (López \& Hernández, 2009, p. 54)

De esta manera se visualiza cómo la riqueza hídrica y de cobertura vegetal potencian al corregimiento de Tribunas Córcega como un área de interés especial para la conservación y estudio de la historia natural de las aves rapaces, aves migratorias y aves bajo algún grado de amenaza y cómo sus características ecológicas han contribuido a ser un remanente que contribuye a la conservación de estas especies.

El trayecto de Esperanza -Galicia, Parque Recreacional Comfamiliar Galicia se caracteriza por:

Poseer bosques muy cerca de la transición hacia los bosques submontanos. La pluviosidad, que oscila entre 1500 y $1800 \mathrm{~mm}$, está repartida en dos épocas, de abril a mayo y de octubre a noviembre, en las que cae el $70 \%$ de la lluvia total anual; la sequía en estos bosques no parece ser tan severa como en otras regiones, pues el período en que la 


\begin{abstract}
vegetación pierde el follaje no suele prolongarse por más de unas pocas semanas. En los parches mejor conservados, como en el de Las Pilas, cerca de la ciudad de Cartago, la vegetación arbórea alcanza un dosel de $30 \mathrm{~m}$, con algunos elementos prominentes de caracolí, burilico, manteco, higuerón, yarumo y ceiba. Una particularidad especial de estos bosques es que frecuentemente aparecen entremezclados con guaduales (Banco de Occidente, 2006)
\end{abstract}

Convirtiendo a este sector en uno de los mejores relictos más amenazado de los ecosistemas del país y explicando porqué a pesar de la reducción general y deterioro que ha tenido este ecosistema en el país, sigue siendo un punto importante de conservación de las aves dentro del área de estudio, haciéndose más urgente la necesidad de preservarlo contribuyendo a la preservación de la avifauna asociada.

\title{
AVES RAPACES (CATHARTIDAE, PANDIONIDAE, ACCIPITRIDAE Y FALCONIDAE)
}

Son varios los elementos que se deben tener en cuenta para tratar de justificar la presencia de aves rapaces (para este caso dícese de las aves pertenecientes a las familias Cathartidae, Pandionidae, Accipitridae y Falconidae) dentro del área de estudio.

Para Márquez et al.(2005, pp.37-38):

La mayor diversidad en cuanto a número de especies [...] de aves rapaces diurnas de Colombia [...] está en la región Norandina (desde la serranía de Perijá a lo largo de las tres cordilleras hacia el sur hasta el departamento de Nariño).

A la vez existen unos cuantos géneros que se encuentran representados en la mayoría de las regiones (según la caracterización por "provincias biogeografías" presentada por Márquez et al., 2005, p. 31) como Accipiter, Buteo y Falco. En cuanto a las especies, Cathartes aura y Coragyps atratus son las especies de distribución más amplia, y la presencia de Pandion haliaetus y Buteo platypterus, puede deberse a que son migratorias. "Estas especies se caracterizan por tener requerimientos de hábitats poco restringidos (hábitats boscosos, acuáticos y áreas abiertas), como el caso de Cathartes aura y Coragyps atratus" (Márquez et al.,2005, p. 38).

Según McMullan et al. (2010, p.IX) la ciudad de Pereira se encuentra ubicada dentro de los ecosistemas de bosque húmedo tropical, piedemonte y subtropical, pero los cambios en 
el uso del suelo a causa del fenómeno de la urbanización han generado altas perturbaciones en estos ecosistemas. Aun así "debemos tener en cuenta también que estos ecosistemas son el resultado de los contrastes regionales del relieve (persistentes hoy día) y que por ello aun pueden encontrarse en una misma provincia una gran diversidad de ecosistemas, incluso en áreas aparentemente homogéneas" (Márquez et al., 2005, p. 41) que sustenten poblaciones de aves silvestres como las rapaces. También es importante considerar que "la distribución actual de rapaces en Colombia es el resultado de una compleja red de interacciones climáticas, geomorfológicas y ecológicas" (Márquez et al., 2005, p.41) no dependiendo su presencia estrictamente solo de la calidad de conservación del ecosistema original ni de su grado de intervención antrópica, ni siquiera dentro de asentamientos humanos tan alterados como lo son las urbes.

Solo unas cuantas especies de rapaces están presentes en hábitats muy específicos o son demográficamente raras (según registros) aunque su área de distribución sea amplia y no estén asociados a hábitats muy específicos. "La gran mayoría de las especies habitan bosques bajos tropicales, bosques deciduos, bosques de galería y bosques montanos siempre verdes" (Márquez et al., 2005, p.42). "Este hecho también se ve evidenciado parcialmente por la distribución altitudinal; la mayor concentración de especies de rapaces (aproximadamente el $70 \%$ de las especies) caen dentro del rango entre el nivel del mar y los $1500 \mathrm{~m}$ de altura" (Márquez et al., 2005, p. 42).Estando la ciudad de Pereira dentro de este rango (1411 msnm) correspondiendo a los bosques premontanos y montanos bajos en la región subandina. Márquez et al. (2005, p.43) afirman que en el territorio colombiano, la diversidad biológica se concentra principalmente en el área del piedemonte y en las estribaciones interiores de las cordilleras. Así mismo afirma que es de esperarse que la riqueza de especies disminuya con la altura, encontrándose una disminución hacia los $1500 \mathrm{~m}$. Con respecto a las familias, la riqueza de halcones (Falconidae) decrece más rápidamente que la de las águilas y gavilanes (Accipitridae) con la altitud, debido a que a mayor altura hay simplificación de los hábitats y quizás menor disponibilidad de los mismos. Esto podría justificar la presencia de 7 especies de la familia Accipitridae con respecto a solo 4 de la familia Falconidae.

Aunque muchas rapaces dependen de bosque primario para buscar alimento y anidar, algunas especies pueden sobrevivir en grandes áreas transformadas en mosaicos de diferentes y cambiantes tipos de vegetación secundaria, o inclusive en zonas de cultivos, pastos o tierras desnudas expuestas a la erosión (Márquez et al., 2005, p. 49)

Este es un elemento que puede argumentar la presencia de aves rapaces dentro de áreas urbanas (Tabla 3), así las actividades antrópicas que crean claros, pastizales, áreas abiertas con arbustos y árboles dispersos, jardines y áreas 
cultivadas favorecen a ciertas especies de Accipitriformes aumentando su densidad según Márquez et al. (2005). Los requerimientos ecológicos intrínsecos de las Falconiformes hacen que muchas de estas aves puedan ser consideradas como excelentes bioindicadores, a través de las cuales se puede evaluar y monitorear los esfuerzos de conservación en diferentes ecosistemas, señalando en específico la importancia de la conservación de zonas verdes dentro y en la periferia de la ciudad, en definitiva es clave la presencia de parches verdes. Muchos consideran que pequeñas zonas verdes no son suficientes para sostener poblaciones de aves silvestres, tal vez sea así, pero el observar estas especies dentro de la ciudad sugiere que es importante conservar estas áreas así como ampliarlas y establecer nuevas, que no solo serían pulmones de la ciudad sino también refugios de los reductos de poblaciones de fauna silvestre que originalmente se distribuyeron allí, "aun así las relaciones entre la calidad de hábitat y el estado de las poblaciones de algunas especies de zonas abiertas, no son lo suficientemente claros como para asegurar si estas especies están aumentando o disminuyendo" (Márquez et al., 2005, p.55).

Tabla 3. Estado de conservación, dependencia a bosques y distribución tropical, de las aves rapaces presentes en Pereira

\begin{tabular}{|c|c|c|c|c|}
\hline \multirow{2}{*}{ Especies } & \multicolumn{2}{|c|}{ Estado de conservación } & \multirow{2}{*}{$\begin{array}{c}\text { Dependencia de } \\
\text { bosques }\end{array}$} & \multirow{2}{*}{$\begin{array}{l}\text { Distribución } \\
\text { tropical }\end{array}$} \\
\hline & Global & Nacional & & \\
\hline \multicolumn{5}{|c|}{ CATHARTIDAE: (2) (Gallinazos o Buitres Americanos) } \\
\hline Cathartes aura & $\mathrm{Na}$ & $\mathrm{Na}$ & No & $\mathrm{P}$ \\
\hline Coragyps atratus & $\mathrm{Na}$ & $\mathrm{Na}$ & No & $P$ \\
\hline \multicolumn{5}{|c|}{ PANDIONIDAE: (1) (Aguila Pescadora) } \\
\hline Pandion haliaetus & $\mathrm{Na}$ & $\mathrm{Na}$ & No & $P$ \\
\hline \multicolumn{5}{|c|}{ ACCIPITRIDAE: (7) (Gavilanes, Aguilas y Aguilillas) } \\
\hline Elanus leucorus & $\mathrm{Na}$ & $\mathrm{Na}$ & No & $\mathrm{P}$ \\
\hline Ictinia plumbea & $\mathrm{Na}$ & $\mathrm{Na}$ & No & $P$ \\
\hline Accipiter striatus & $\mathrm{Na}$ & $\mathrm{Na}$ & No & $P$ \\
\hline Accipiter bicolor & $\mathrm{Na}$ & $\mathrm{Na}$ & No & $\mathrm{P}$ \\
\hline Buteo magnirostris & $\mathrm{Na}$ & $\mathrm{Na}$ & No & $P$ \\
\hline Buteo platypterus & $\mathrm{Na}$ & $\mathrm{Na}$ & Si & $\mathrm{P}$ \\
\hline Buteo albicaudatus & $\mathrm{Na}$ & $\mathrm{Na}$ & No & $P$ \\
\hline \multicolumn{5}{|c|}{ FALCONIDAE: (4) (Halcones, Caracaras) } \\
\hline Caracara cheriway & $\mathrm{Na}$ & $\mathrm{Na}$ & No & $P$ \\
\hline Mivago chimachima & $\mathrm{Na}$ & $\mathrm{Na}$ & No & $P$ \\
\hline Falco sparverius & $\mathrm{Na}$ & $\mathrm{Na}$ & No & No tropical \\
\hline Faico peregrinus & $\mathrm{Na}$ & $\mathrm{Na}$ & No & No tropical \\
\hline
\end{tabular}

El Gallinazo Negro (Coragyps atratus) es una especie común y localmente abundante en toda Colombia, generalmente asociada a áreas abiertas y semiabiertas, siendo más numerosa en los alrededores de las ciudades principalmente en basureros o rellenos sanitarios o en ocasiones en los bordes de carreteras en donde se arrojan basuras. Es poco común en áreas de bosques primarios y poco fragmentados. El Gavilán Maromero (Elanus leucurus) es una especie adaptada a espacios urbanos y suburbanos, la cual ha aumentado en las últimas décadas en este tipo de hábitat beneficiándose de la apertura de claros y deforestación. La Aguililla Plomiza (Ictinia 
plumbea) es una especie gregaria de vuelo grácil y sostenido que habita en mosaicos de parches de bosque y zonas abiertas. El Azor Cordillerano (Accipiter striatus) se puede ver en áreas abiertas. El Azor Bicolor (Accipiter bicolor):

No está asociado a algún hábitat en particular, en la Guyana se ha reportado en bosque húmedo, en otros países es más común en bosques más secos, abiertos y delgados, además ensabanas con bosques de galería y parches de bosque aislados, y en bosques secundarios (Grossman \& Hamlet, 1964 y Brown \& Amadon, 1968 citados en Márquez et al., 2005, p. 168)

El Gavilán Caminero (Buteo magnirostris) (Figura 2) es el gavilán más común de todos, presenta poblaciones suburbanas. El Gavilán Coliblanco (Buteo albicaudatus) se encuentra en zonas tropicales y subtropicales, usualmente en campos abiertos y semiáridos, aunque también están en arbolados secos y abiertos, y sabanas húmedas con palmas. El Caracara Moñudo (Caracara cheriway):

Se encuentra en campos abiertos y semiabiertos; potreros, sabanas con palmeras esparcidas, bordes de río y especialmente haciendas (en los llanos), algunas veces en zonas boscosas y humedales. Principalmente en tierras bajas, pero ha ido incrementando su presencia en zonas altas de Colombia y Ecuador (del Hoyo et al., 1994 citados en Márquez et al., 2005, p. 253)

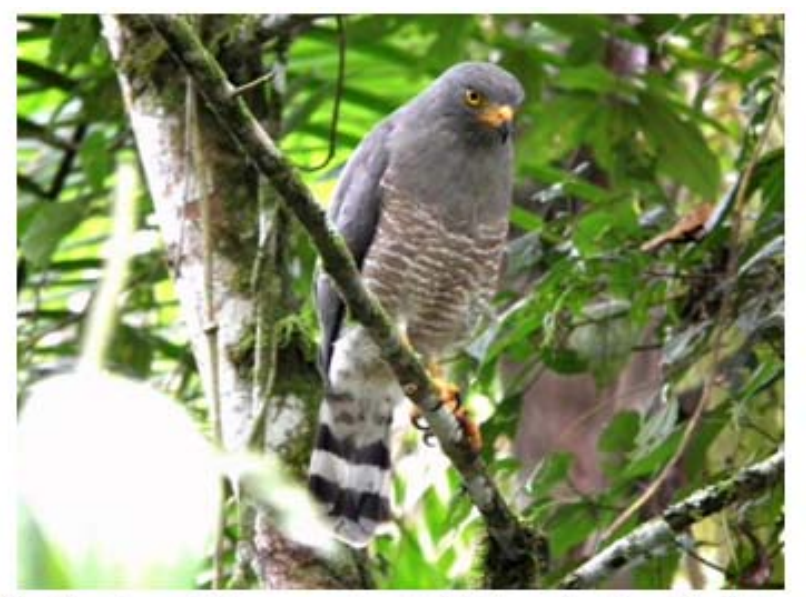

Figura 2. Gavilán Caminero (Buteo magnirostris). Fotografia: Manakin Nature Tours, 2011.

La Pigua (Milvago chimachima)se encuentra en campos abiertos con árboles dispersos, haciendas, potreros, sabanas con palmas, bordes de bosque y a lo largo de los ríos, registrado hasta los $1800 \mathrm{~m}$ en Colombia. El Cernícalo Americano (Falco sparverius): 
Ocupa la mayoría de hábitats, desde las tierras bajas tropicales y los desiertos (aun bajo el nivel del mar) hasta la línea de árboles, a 3700 m en Norteamérica y 4300 m en Suramérica; igualmente ocupa centros urbanos. Común en terrenos alterados, perturbados y en campos agrícolas (del Hoyo et al.,1994 citados en Márquez et al., 2005, p. 324)

Dentro del área de estudio se identificaron 4 especies de aves rapaces migratorias que son Cathartes aura, Pandion haliaetus, Buteo platypterus y Falco peregrinus (Tabla 2). Para entender su presencia dentro del área de estudio se deben tener en cuenta diversos aspectos.

La posición geográfica de Colombia permite que el mayor porcentaje de rapaces migratorias entre a Suramérica por este país luego de haber seguido las rutas por Centroamérica y el Caribe; adicionalmente, para varias especies de rapaces, representa el primer sitio de descanso, para alimentarse o como destino final. En Colombia existen 43 especies de rapaces que se cree que migran en alguna proporción dentro de su rango. Para las especies neárticas, el país proporciona la posibilidad de hacer paradas para descansar y alimentarse antes de continuar hacia sus destinos finales (Márquez et al., 2005, p. 64)

Las cadenas montañosas, como en la que se encuentra el área de estudio, así como lugares "áridos", como las amplias áreas desnudas que ofrecen las urbes, son puntos geográficos frecuentes dentro de las rutas migratorias de rapaces, según Márquez et al. (2005) es probable que la razón de estas congregaciones de rapaces se deba a condiciones favorables en estos sitios, hábitats en áreas contiguas, que son puntos de referencia geográficos utilizados como herramientas de orientación o una combinación de estos. Cantidades adecuadas de agua y alimento son necesarias para cubrir todo el trayecto de migración, por lo cual, estas aves escogen vías cortas, que demanden poco gasto energético y en las que puedan hacer paradas estratégicas para encontrar alimento y disponibilidad de perchas.

Respecto a cada especie:

La mayoría de los individuos de Gavilán Aliancho (Buteo platypterus), utilizan la ruta centroamericana. El rango no reproductivo de esta especie, se extiende desde Costa Rica hasta el norte de Suramérica en la Amazonia. Esta especie se puede observar en parvadas de miles de individuos en su migración por Colombia donde habita bordes de bosque, bosques en crecimiento secundario con claros. 
Sus áreas de hibernación comienzan desde el sur de México hasta los bosques amazónicos. Suele escoger piedemontes, zonas urbanas y suburbanas (inclusive bosques de Eucaliptos) como destino final de su migración (Márquez et al., 2005, p. 66)

En el neotrópico la Guala Cabecirroja (Cathartes aura) es el buitre más grande que migra, sus rangos reproductivos y noreproductivos son continuos y la especie se puede encontrar desde Canadá hasta Tierra del Fuego, habita principalmente en bosques deciduos y zonas cercanas a cultivos y otras áreas abiertas (Márquez et al., 2005, p. 66)

Observaciones de Halcón Peregrino (Falco peregrinus), vive en campos semiabiertos, en todas las zonas climáticas (es menos frecuentemente en tundras y desiertos). Entre las rapaces migratorias que llegan a Colombia, el halcón peregrino es una de las especies que utiliza zonas urbanas. En Bogotá se ha visto en humedales, edificios y antenas de comunicaciones. En el Valle del Cauca durante un sobrevuelo de 45 minutos se encontraron 12 halcones peregrinos posados sobre torres de transmisión eléctrica (Ospina com. per., citado en Márquez et al., 2005, p. 341)

Los individuos de Águila Pescadora (Pandion haliaetus) son una especie asociada al agua y exceptuando la época de migración siempre se encuentra en estuarios, ríos, lagunas y cuerpos de agua tanto naturales como artificiales (Hilty \& Brown, 1986 citados en Márquez et al., 2005, p. 99)

De esta última especie "se conoció que el rango noreproductivo es muy amplío en Suramérica y llega hasta Argentina" (Márquez et al., 2005, p. 99).

\section{AVES MIGRATORIAS}

Dentro de la investigación se catalogaron veintiséis (26) especies de aves migratorias boreales y australes (Tabla 2). A excepción de tres especies consideradas raras que son: Zorzal Rojizo (Catharus fuscescens), Piranga Alinegra (Piranga olivacea) y Reinita Alidorada (Vermivora chrysoptera), todas las demás especies son consideras comunes o bastantes comunes y la mayoría frecuenta áreas abiertas y semiabiertas, bosques y bordes de bosque, granjas y plantaciones, parques, matorrales, senderos, en el suelo algunos y en el dosel casi todos. La mayoría se mueve en bandas mixtas y algunas especies como 
Verderón Ojirrojo (Vireo olivaceus) y Golondrina Blanquiazul (Pygochelidon cyanoleuca cyanoleuca) tienen poblaciones residentes.

"Estudios muy serios sobre las migraciones nos dicen que las especies tienen preferencias por los lugares más cercanos que encuentran en su viajes y los menos competidos por las especies nativas" (Jaramillo, 1993, p. 28). Dentro de los asentamientos humanos como las urbes, las poblaciones de aves silvestres nativas son menores y así mismo podría ser la competencia por disponibilidad de recursos, además el incremento del conflicto entre las aves migratorias de todo tipo y las actividades humanas como la agricultura y autopistas puede empujarlas hacia las ciudades "aparentemente en respuesta a los cambios de recursos alimenticios" (Hilty \& Brown,1986, p. 29).

Los sitios escogidos para detenerse y permanecer son aquellos en los que se hacen esfuerzos por mantener el hábitat en la mejor forma posible. Por supuesto preferirán lugares tranquilos con bosque, agua y comida. ¿Cuál es el indicador que les hace tomar la decisión sobre este o aquel lugar? Indudablemente hay un factor que cuenta y es la presencia de aves nativas en el sitio (Jaramillo, 1993, p. 28)

Dentro del área de la investigación existen amplias zonas con vegetación multiestrato en diferentes estados sucesionales, que pueden por lo menos servir de área de descanso en su paso a zonas menos intervenidas y en mayor estado de conservación dentro del territorio nacional y hacia el sur o el norte como "el oriente colombiano, especialmente los Llanos, lugar ideal para las especies migratorias" (Jaramillo, 1993, p. 28). Así mismo dentro del inventario elaborado para el área de estudio se registraron 178 especies de aves nativas, lo cual podría considerarse como un indicador de que es un área que puede por lo menos sostener a una población de aves que va de paso dentro de su ruta migratoria (Tabla 2).

Con el crecimiento de las ciudades a nivel mundial, las aves migratorias terrestres deben pasar cada vez más a través de amplias zonas urbanas que les ofrecen muy poco del hábitat natural del cual estas especies dependían para recargar sus energías y poder continuar su travesía. Sin embargo, algunas especies han logrado adaptarse a pequeños hábitats urbanos, donde las aves parecen encontrar suficiente alimento (Cisneros, 2010)

Un estudio de caso del Zorzal Buchipecoso o Zorzal de Swainson (Catharus ustulatus) llevado a cabo por Matthews \& Rodewald (2010) refleja "la importancia de estos pequeños remanentes de vegetación [dentro de las áreas urbanas] para las aves revelando que incluso pequeños parches de bosque 
urbano son muy valiosos para las aves migratorias". Dado que "parecen proveer una adecuada protección y alimento para algunas especies de aves migratorias" (Cisneros,2010). Matthews \& Rodewald (2010) indicaron que:

El hecho de que la mayoría de aves hayan permanecido, incluso en las zonas más pequeñas, sugiere que de cierta manera los zorzales de Swainson son flexibles a sus necesidades de hábitat y eran capaces de encontrar sus requerimientos de "parada" dentro de estos parches de bosque Urbanos (Matthews \& Rodewald, 2010)

Este estudio reveló que casi todos los tamaños de bosque parecieron adecuados para los zorzales, sin embargo ellos prefieren zonas más extensas de bosque. Las aves que estuvieron en bosques urbanos más grandes preferían quedarse en el interior en lugar de acercarse a los bordes del bosque y se movían más (en comparación con aquellas en pequeñas áreas) lo que indica que su menor restricción era la búsqueda de comida. Los investigadores afirmaron que este estudio fue únicamente realizado en una especie, por lo que es difícil decir si los resultados son extrapolables a otras especies. Pero, El Zorzal de Swainson es una de las especies más sensitivas en relación al bosque, por lo que su conformidad con pequeños fragmentos de bosque es alentadora (Cisneros, 2010)

Los remanentes de bosque dentro de zonas urbanas poseen valor para las Naciones Unidas en la conservación de las aves terrestres migratorias. Obviamente, los parches de mejor tamaño, pero incluso los de menor tamaño valen la pena conservados. Las áreas verdes urbanas, por más grandes que sean, no pueden reemplazar los bosques nativos, pero son de gran ayuda para muchas aves que en caso contrario desaparecerían al no encontrar comida y refugio (Cisneros, 2010)

\section{AVES BAJO ALGÚN GRADO DE AMENAZA}

Se identificaron siete (7) especies bajo algún grado de amenaza según McMullan, Donegan y Quevedo (2010) y Renjifo et al. (2002) (Tabla 2).

Penelope perspicax es una especie endémica de Colombia. La especie está considerada a nivel mundial como en peligro (EN). Esta pava 
ha perdido el $95 \%$ de su hábitat natural. Posiblemente más de un $30 \%$ de esta pérdida haya ocurrido en tres generaciones. Adicionalmente la especie está sometida a la presión de la cacería (Renjifo et al., 2002, p. 124)

Se encuentra en el oriente de la ciudad dentro del área del S.F.F. Otún-Quimbaya y su área de influencia, su población se ha recuperado "ya que durante los años cuarenta y cincuenta la cobertura de bosque de la región fue más reducida que en la actualidad" (Renjifo et al., 2002, p. 128), gracias a la conservación y "la recuperación de la cobertura de bosque y protección de la cacería" (Renjifo et al., 2002, p. 128). La única otra especie miembro de la familia Cracidae presente en el área de estudio bajo algún grado de amenaza es Aburria aburri la cual presenta una situación similar a la anterior especie.

Chlorochrysa nitidissima (Figura 3) y Dacnis hartlaubison consideradas como en estado Vulnerable (VU) por Renjifo et al.(2002, p. 415) y McMullan et al.(2010, p. 195), ambas son especies endémicas de Colombia. C. nitidissima es una especie considerada "local y rara presente en los ecosistemas de bosque húmedo tropical, piedemonte y subtropical; en bandadas mixtas" (McMullan, Donegan y Quevedo, 2010, p. 195). Tal vez C. nitidissima se encuentre dentro del área de estudio empujada por la pérdida de su hábitat natural a causa de la deforestación y viéndose obligada a establecerse en las zonas verdes del perímetro urbano donde puede encontrar alimento y refugio.

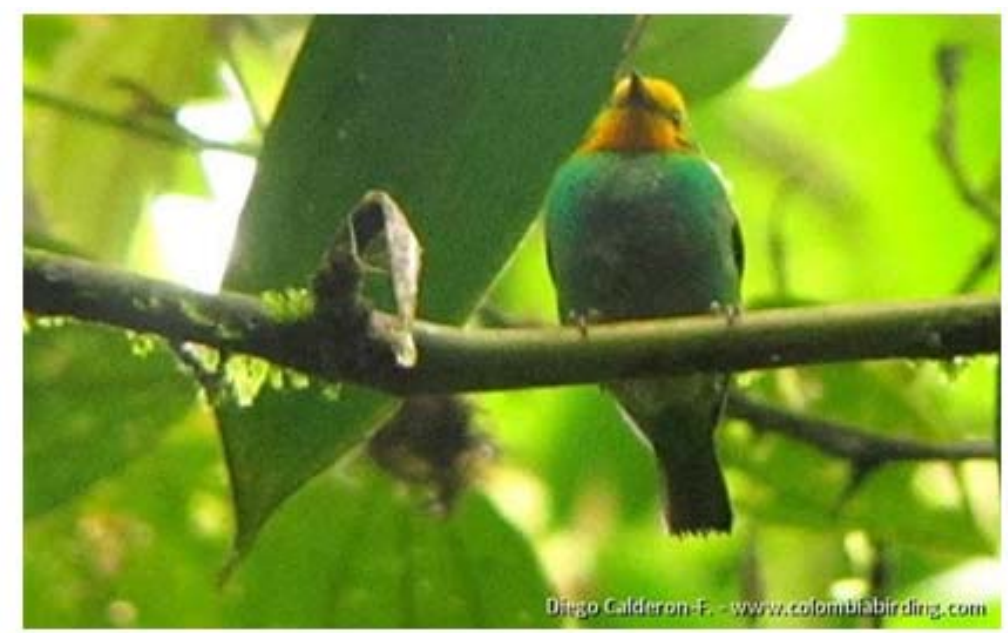

Figura 3. Tángara Multicolor (Chlorochrysa nitidissima). Fotografia: Diego Calderón Colombia Birding, 2011.

Dacnis hartlaubi "es una especie endémica de Colombia y rara" (Stiles, 1998a; Hilty \& Brown, 1986 y Botero \& Verhelst, 2001 citados en Renjifo et al., 2002, p. 420), "con una distribución aparentemente fragmentada y restringida" (Hilty \& Brown, 1986, p. 607; McMullan, Donegan y Quevedo, 2010, p. 199)."La especie utiliza generalmente el dosel y subdosel del bosque, 
donde se le ha visto en grupos de dos o tres individuos o acompañando a bandadas mixtas de especies residentes y migratorias" (Hilty \& Brown, 1986; Kaestnerin litt., 1992 y Botero \& Verhelst obs. per., 2000 citados en Renjifo et al., 2002, p. 422). "La mayoría de registros de este dacnis están entre los 1300 y $2200 \mathrm{~m}$, aunque existen registros hasta 2845 y desde los 300 m" (Hilty \& Brown, 1986; Collar et al., 1992 y Wege \& Long, 1995 citados en Renjifo et al., 2002, p. 422). "El Dacnis Turquesa ha sido reportado como habitante del bosque húmedo, de bordes de bosques y claros con árboles esparcidos" (Munves, 1975; Ridgely \& Tudor, 1989 y Stiles \& Bohórquez, 2000 citados en Renjifo et al., 2002, p. 422) y en cafetales con sombrío, adyacentes a parches de bosque y rastrojo (Collar et al., 1992; Botero \& Verhelst obs. per., 2000 citados en Renjifo et al., 2002, p. 422). Según McMullan, Donegan y Quevedo(2010, p. 199) la especie está presente en un rango altitudinal comprendido entre los 1000-2200 m en bordes de bosque locales y plantaciones subtropicales de los Andes centrales y orientales, no en grupos mixtos. Dentro del área de estudio ha sido registrada por Arias et al. (2008) y Londoño (2011) en el campus y Jardín Botánico de la Universidad Tecnológica de Pereira a 1470 msnm (Tabla 1). Esta especie ha sido clasificada como vulnerable (Collar et al., 1992 y Renjifo, 1998 citados en Renjifo et al., 2002, p. 423; McMullan, Donegan y Quevedo, 2010, p. 199) debido a que su población total es supuestamente baja y está decreciendo rápidamente y a que su distribución es pequeña y fragmentada. "Las poblaciones pueden estar disminuyendo debido a la magnitud de la deforestación en las partes bajas de los Andes en donde la especie ha sido encontrada" (Ridgely \&Tudor, 1989 citados en Renjifo et al., 2002, p. 423). "Este dacnis ha perdido un $73 \%$ de su hábitat" (Renjifo et al., 2002, p. 424). Su presencia dentro del área de estudio puede ser justificada teniendo en cuenta que el área donde ha sido observada ha sido consagrada como un área de protección, donde se ha velado por la recuperación de la flora nativa y conserva los requerimientos de su hábitat anteriormente descritos(Tabla 1).Finalmente Arias et al. (2008, p. 16) consideran que, al parecer, en los últimos años ha ampliado su rango de distribución al norte de los Andes, poblando zonas donde antes no se había registrado como el Eje Cafetero y en particular en relictos boscosos del área urbana del municipio de Pereira.

Saltator cinctus es una especie considerada por Renjifo et al. (2002, p. 452) como vulnerable (VU).

Esta especie ha perdido el $54 \%$ de su hábitat, y debido a la aceleración de la pérdida de hábitat en su rango de distribución por causa de los cultivos ilícitos, se estima que perderá más de un $30 \%$ de su hábitat y por lo tanto de su población en 10 años. Se sabe que esta especie tiene una distribución localizada dentro de su hábitat potencial, y se estima que el área de ocupación puede ser de menos de la mitad de su hábitat potencial, lo que hace de esta una especie vulnerable (Renjifo et al., 2002, p. 452) 
Pero para McMullan, Donegan y Quevedo (2010, p. 203) se considera como casi amenazado (NT) así como para Birdlife International (2000 citado en Renjifo et al., 2002, p. 452), "rara y local" (Uribe, 1986 y Renjifo,1991a citados en Renjifo et al., 2002, p. 452).

Ha sido reportado en los bosques andinos y subandinos en ambas vertientes de la cordillera Central desde el Valle del Cauca, Tolima hasta Caldas, entre 1800 y 3080 m de altitud, es una especie de bosques andinos que ocasionalmente baja al cinturón subandino (Renjifo et al., 2002, p. 450)

Se encuentra en bajas densidades poblacionales en bosque maduro, así como en bosque secundarios, silvicultivos de aliso (Alnus acuminata) y bordes de bosque con o sin Chusquea (Renjifo, 1991a; Verhelstet al. com. pers., 2000; Kattan \& Beltran com. pers., 2000 y Quevedo in litt., citados en Renjifo et al., 2002, p. 450). Se encuentra amenazada por la deforestación de su hábitat. Se encuentra dentro del área de estudio dado que el corregimiento de Tribunas - Córcega (Tabla 1), donde fue observada posee ecosistemas de bosque secundario en diversos estados sucesionales (bosque de niebla) según sus requerimientos de hábitat y alimentación.

Vermivora chrysoptera considerada como casi amenazada (NT) por McMullan, Donegan y Quevedo (2010, p. 213) y como una rara especie migratoria boreal en bandadas mixtas de dosel. Su amenaza se deba tal vez como en otras especies a la pérdida de su hábitat. Esta especie ha sido observada dentro del área de estudio dentro del campus de la Universidad Tecnológica de Pereira y su Jardín Botánico, el cual como ha sido referenciado ya anteriormentees una zona con abundantes relictos boscosos poco intervenidos por actividades antrópicas o que han sido abandonados permitiendo la recuperación de la cobertura vegetal en multiestratos; el campus de la Universidad Tecnológica y su jardín botánico se caracterizan por poseer ecosistemas de bosque de guadua, bosque secundario en diferentes estados sucesionales (bosque andino) y un humedal, donde puede satisfacer sus requerimientos de hábitat y alimenticios mientras nos visita. Es la única especie que no se encuentra simultáneamente referenciada por Renjifo et al. (2002), quizás esto se deba a que la presión por la pérdida de su hábitat se ha incrementado en los últimos nueve años.

Hypopyrrhus pyrohypogaster es una especie endémica, catalogada como en peligro (EN) tanto para Renjifo et al. (2002, p. 468) como para McMullan, Donegan y Quevedo (2010, p. 221), siendo la categoría más grave entre las especies aquí referenciadas, considerada "local y rara en bosques de niebla, usualmente en el dosel, con bandadas mixtas de arrendajos y otras grandes aves, su rango altitudinal está comprendido entre los 1200-3000 m" (McMullan, Donegan y Quevedo, 2010, p. 221). Su presencia puede argumentarse por algunos relictos de bosque de niebla secundarios en zonas dentro del área de 
investigación como el corregimiento de Tribunas -Córcega y el Jardín Botánico de la Universidad Tecnológica de Pereira.

La principal amenaza para las aves es la destrucción de su hábitat, que es causado en gran medida por el desarrollo urbano. A nivel mundial, las causas de esa destrucción son: el crecimiento de las ciudades y las obras de infraestructura requeridas por las ciudades, como las carreteras que conectan los centros urbanos, las represas que abastecen de agua a las ciudades y las hidroeléctricas que producen energía para las ciudades, que ponen en riesgo a 350 especies. La agricultura y la ganadería extensivas -requeridas para alimentar a los habitantes de las ciudades-, que amenaza a 800 especies. La extracción minera -para producir materiales de construcción, para producir energía para las ciudades, o para que la industria genere los productos demandados por ellas- que pone en riesgo a 600 especies. Además, la contaminación del suelo (rellenos sanitarios) y la contaminación de las aguas (vertimientos industriales, alcantarillados) amenazan a 120 especies; y la polución del aire (automotores, industrias) amenaza a 30 más (BirdLife, 2002, p. 7 y BirdLife, 2004, pp. 50-53 citados en Osorio \& Molina, 2009, pp. 4950)

Es evidente que el impacto ejercido por las ciudades sobre las aves del mundo es devastador; no obstante y aunque resulte paradójico, una de cada cinco especies amenazadas se refugia en ambientes urbanos o rurales totalmente artificiales (BirdLife, 2002); especialmente en los árboles y las rondas hídricas, donde pueden anidar y alimentarse. En otras palabras, el $20 \%$ de las aves amenazadas o desplazadas de su hábitat natural, se refugian en las ciudades o en torno a ellas. De manera que desde la arquitectura y el urbanismo se puede contribuir en la conservación de muchas especies de aves, algunas de ellas en riesgo de extinción (Osorio \& Molina, 2009, p. 50)

Al fin y al cabo la mayoría de las especies referenciadas aquí bajo algún grado de amenaza (amenaza que se debe principalmente a la destrucción de su hábitat) son especies consideradas de por sí como raras y locales y su presencia se debe a que, así sea por muy poco, su rango de distribución abarca el área de estudio. 


\section{ESPECIES ENDÉMICAS}

Se registraron cuatro (4) especies endémicas. "Las especies restringidas espacialmente son buenas indicadoras porque representan tipos de hábitats específicos" (Ribera, 1991 citado en Villegas \& Garitano, 2008, p. 151), por ejemplo el Diamante Emperador (Heliodoxa imperatrix) es la única de las cuatro especies endémicas registradas dentro del área de estudio considerada como rara. Las otras tres especies son comunes, aunque requieren hábitats distintos, por ejemplo la Batará Carcajada(Thamnophilus multistriatus)habita bosques secos y matorrales propios del trayecto1 (Esperanza-Galicia, Parque Recreacional Comfamiliar Galicia); el Atrapamoscas Pechirrufo (Leptopogon rufipectus)en bosque subtropicales como los de los trayectos 2 (corregimiento Tribunas -Córcega), 3 (campus y Jardín Botánico de la Universidad Tecnológica de Pereira), y 5 (barrio El Plumón, vía de acceso Aeropuerto Internacional Matecaña y Zoológico Matecaña); y el Toche Enjalmado (Ramphocelus flammigerus) (Figura 4) en bordes de bosque y claros, plantaciones como el trayecto 6 (comuna San Joaquín) (Tabla 1). Tal vez su presencia dentro del área de investigación se deba a que en conjunto en la ciudad existen relictos de los diversos tipos de bosque habitados por estas especies y en el caso de $R$. flammigerus el cual también habita plantaciones, ecosistemas que circundan la ciudad.

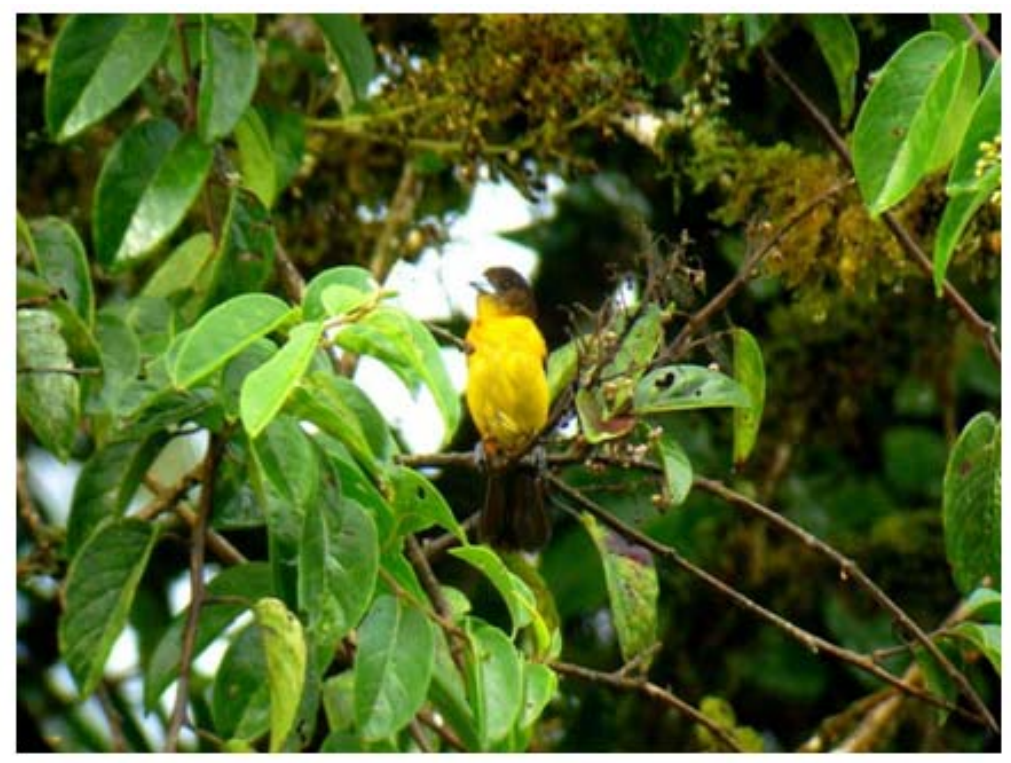

Figura 4. Toche Enjalmado $\varrho$ (Ramphocelus flammigerus). Fotografia: Manakin Nature Tours, 2012

La diversidad de hábitats es un factor importante en el mantenimiento de la biodiversidad a nivel regional, y sin referencias explícitas de los requerimientos de hábitat de cada especie es difícil establecer criterios que permitan su conservación (Hansem et al., 1993 citados en Serrano \& Aparicio,1996). Es necesario considerar dos factores en el momento de analizar la importancia de la diversidad de hábitats: 1) La riqueza de especies aportadas. Las especies 
más vulnerables, es decir, las especialistas de hábitat o las especies de rangos geográficos restringidos, están probablemente concentradas en los hábitats/ecosistemas más complejos, mientras que las especies invasoras y de rangos geográficos amplios habitan ecosistemas sencillos, 2) por otra parte, la configuración del paisaje representada en las proporciones de las distintos hábitats y su conectividad determinan la posibilidad de mantener metapoblaciones en flujo de individuos entre unidades de hábitat y paisajes (Serrano\& Aparicio,1996).

\section{CONCLUSIONES}

Es probable que la mayoría, tal vez algunas y no todas de las especies aquí referenciadas, sean especies "generalistas" o la plasticidad del nicho les permite adaptarse a ambientes con características más o menos similares a las que requieren según sus condiciones. El encontrar especies de aves rapaces dentro del área de estudio puede deberse a que la mayor diversidad en cuanto a número de especies de aves rapaces diurnas de Colombia está en la región Norandina, y a que las especies presentes se caracterizan por tener requerimientos de hábitats poco restringidos (hábitats boscosos, acuáticos y áreas abiertas). Las especies migratorias prefieren áreas menos competidas por las especies nativas, como lo son las zonas urbanas y tal vez son flexibles a sus necesidades de hábitat, haciéndolas capaces de encontrar sus requerimientos de "parada" dentro de estos parches de bosque urbanos. Las especies bajo algún grado de amenaza encuentran refugio dentro de las áreas naturales periféricas a la ciudad, así como las zonas circundantes a estas, además estas especies son consideradas raras por naturaleza y su amenaza es por destrucción de hábitat principalmente. De las cuatro especies de aves endémicas registradas solo una es considerada rara, las demás son comunes y en términos generales en la ciudad existen relictos de los diversos tipos de bosque requeridos por estas especies.

Es posible conservar una alta proporción de la avifauna nativa y exótica silvestre (en este caso las especies migratorias) en paisajes fragmentados como las zonas urbanas, preservando y recuperando la diversidad de hábitats naturales. Las aves pueden indicarnos ciertas características del hábitat, su presencia o ausencia nos puede ayudar a discernir patrones o umbrales de impactos ambientales, puesto que algunas especies persisten a lo largo de gradientes de disturbio mientras que otras desaparecen. 


\section{RECOMENDACIONES PARA LA CONSERVACIÓN DE LAS POBLACIONES DE AVES RAPACES, AVES MIGRATORIAS Y AVES BAJO ALGÚN GRADO DE AMENAZA EN LA CIUDAD DE PEREIRA (RISARALDA)}

"Las aves sufren varias amenazas, pero sin dudas, la mayor de ellas es la transformación del hábitat. De las aves de todo el mundo que están en riesgo de extinción, el 85\% lo están por esta causa" (Asociación Conservacionista Uruguaya de Ornitología, s.f.). Actividades como "la construcción de obras civiles, transforman los hábitat naturales que se caracterizan por ser complejos y diversos, en hábitat biológicamente degradados e inhóspitos para la mayoría de la vida silvestre" (ACOPAZOA, 2003, p. 87).

La reducción de la cobertura boscosa y de su ecosistema, el aislamiento de las poblaciones en parches de hábitat, la alteración de los procesos de interacción entre especies y la transformación de su entorno, son las principales amenazas para la conservación de las poblaciones de las aves silvestres en los ecosistemas urbanos.

Por ello para contribuir a la conservación de las poblaciones de las aves silvestres se recomienda, en el marco de la Estrategia Nacional para la Conservación de las Aves de Colombia (Renjifo et al., 2000) y el Plan para la Conservación de las Aves Migratorias en Colombia (Fundación ProAves, 2009), adoptar las siguientes medidas para garantizar las conservación de las aves rapaces, aves migratorias y aves bajo algún grado de amenaza de la ciudad de Pereira así como para preservar, mantener o restablecer los biotopos y sus hábitats.

\section{La protección de los hábitats}

La desaparición de los hábitats o su deterioro representa una amenaza para la conservación de las aves silvestres. Por ello, es esencial protegerlos.

Para preservar, mantener o restablecer los biotopos y los hábitats de las aves, la autoridad o autoridades pertinentes deben:

- Designar zonas de protección (santuarios de fauna y flora, parques naturales municipales).

- Mantener y ordenar los hábitats de acuerdo con los imperativos ecológicos.

- Restablecer los biotopos destruidos teniendo en cuenta la flora recomendada en SAO (2009) y crear otros nuevos.

\section{Las zonas de protección especiales}

La autoridad o autoridades pertinentes crearán zonas de protección especialespara las especies de aves amenazadas y las aves migratorias como los santuarios de fauna y flora y los 
parques municipales naturales. Estas zonas deberán encontrarse en el área de distribución natural de las aves así como en la periferia de la ciudad de Pereira y pueden incluir zonas de invernada, nidificación o de descanso en los pasos migratorios.

La autoridad o autoridades pertinentes prestarán especial atención a las zonas húmedas, que se encuentran en regresión en el área de estudio. Deberán asimismo crear unas condiciones favorables para la supervivencia y reproducción de las especies presentes en las zonas de protección especial. Para ello, adoptarán las medidas precisas para evitar la contaminación y el deterioro de los hábitats, así como las perturbaciones que afecten a las aves. También deberán evaluar los impactos de los proyectos susceptibles de tener efectos significativos en los lugares designados y adoptarán las medidas adecuadas para evitar dichos impactos.

Es menester incluir dentro de las zonas de protección las áreas que comprenden los trayectos número 3 (campus y Jardín Botánico de la Universidad Tecnológica de Pereira), número 1 (Esperanza -Galicia, Parque Recreacional Comfamiliar Galicia) y el número2 (corregimiento Tribunas -Córcega) (Tabla 1), dado que son zonas con "alta diversidad de especies " (Londoño, 2011, p. 94) favorecidas por "varios tipos de ecosistemas y fragmentos de bosque interconectados por corredores que dan lugar a áreas con amplia cobertura vegetal en diversos estados sucesionales" (Londoño, 2011, p. 94).

\section{NOTA}

Respecto a las aves rapaces ver el apartado: Aves Rapaces (Cathartidae, Pandionidae, Accipitridae y Falconidae).

\section{BIBLIOGRAFÍA}

- ACOPAZOA -Asociación Colombiana de Parques Zoológicos y Acuarios-. (2003). Biodiversidad. Colombia país de vida. Programa de formación ambiental para maestros. Bogotá: Cargraphics S.A.

- Arias, C.; Rivera, J.; Muñoz, J., Quintero, C. y Berrío, J. (2008). Avifauna representativa Universidad Tecnológica de Pereira - Risaralda - Colombia. Pereira: Semillero de Investigación en Fauna \& Fundación Ambiental WANDRA.

- Asociación Conservacionista Uruguaya de Ornitología. (s.f.). Aves en peligro de extinción. Obtenido el 6 de noviembre de 2012. http://www.avesacuo.com/avesamenazadas.htm

- Banco de Occidente. (2006). Bosque Seco Tropical Colombia. Cali: Banco de Occidente. Obtenido el 9 de 
marzo de $2013 . \quad$ En http://www.imeditores.com/banocc/seco/presentaci on.htm

- Cisneros, H. (2010). Los parques y jardines son importantes para las aves migratorias.http://cisnerosheredia.blogspot.com/2010/08 /los-parques-y-jardines-son-importantes.html

- Calderón, Diego - Colombia Birding. Multicolored Tanager (Chlorochrysa nitidissima) The very same ind in the very same branch, 11 November 2011, Otún Quimbaya Santuary, Pereira, Risaralda Department, Colombia. Imagen tomada del sitio the Internet Bird Collection:

http://ibc.lynxeds.com/photo/multicolored-tanagerchlorochrysa-nitidissima/very-same-ind-very-samebranch (Fecha de actualización 25 de agosto de 2012).

- Faggi, A. y Perepelizin, P. (2006). Riqueza de Aves a lo Largo de un Gradiente de Urbanización en la Ciudad de Buenos Aires. Rev. Mus. Argentino Cienc. Nat., n.s., 8(2), 289-297.

- Fundación ProAves. (2009). Plan para la Conservación de las Aves Migratorias en Colombia. Conservación Colombiana, 11, 1-154.

- Hilty, S. y Brown, W. (1986). A guide to the birds of Colombia. Princeton Un. Press.

- Jaramillo, L. (1993). Aves de Colombia 167 especies... ¡déjelas volar! Santa Fe de Bogotá: ATA Fondo Filantrópico.

- Jardín Botánico Universidad Tecnológica de Pereira. (s.f.). Conservación e Investigación. Obtenido el 6 de noviembre de 2012. http://www.utp.edu.co/jardin/conservamos.html

- Londoño, J. C. (2011). Una Mirada a la Diversidad Ornitológica de Pereira. Bol. Cient. Mus. Hist. Nat. U. de Caldas, 15(1), 84-103.

- López, P. y Hernández, E. (2009). Estudio de riesgo sobre la calidad del agua en la empresa de servicios públicos de Tribunas Córcega - ESPTRI para la formulación de estrategias de gestión ambiental del recurso hídrico - GARH corregimiento Tribunas Córcega - Pereira. Universidad Tecnológica de Pereira.

- Manakin Nature Tours. Roadside Hawk (Bueto magnirostris) A bird perched, 4 March 2011, Otún Quimbaya Santuary, Pereira, Risaralda Department, Colombia. Imagen tomada del sitio the Internet Bird Collection: http://ibc.lynxeds.com/node/176163 (Fecha de actualización 25 de agosto de 2012).

- Manakin Nature Tours. Flame-Rumped Tanager (Ramphocelus flammigerus) A female perched on a tree, 10 April 2012, Otún Quimbaya Santuary, Pereira, Risaralda Department, Colombia. Imagen tomada del sitio the Internet Bird Collection:http://ibc.lynxeds.com/photo/flame-rumpedtanager-ramphocelus-flammigerus/female-perched-tree (Fecha de actualización 25 de agosto de 2012). 
- Márquez, C.; Bechard, M.; Gast, F. y Vanegas, V. H. (2005). Aves rapaces diurnas de Colombia. Bogotá, D.C.: Instituto de Investigación de Recursos Biológicos "Alexander von Humboldt".

- Matthews, S. N. y Rodewald, P. G. (2010a ). Movimiento Comportamiento de un Pájaro del Bosque en un Paisaje Urbanizado: la Importancia Relativa del Nivel de los Efectos del Parche y la Condición Corporal Durante la Parada Migratoria. Ecología del Paisaje. DOI: $\quad$ 10.1007/s10980-010-94750.http://cisnerosheredia.blogspot.com/2010/08/losparques-y-jardines-son-importantes.html

- $\quad$ (2010b ). Parches Bosque Urbano y Duración de la Parada de los Tordos de Swainson Migratorias. El Cóndor, 112(1),96-104.DOI: 10.1525/cond.2010.090049. http://cisnerosheredia.blogspot.com/2010/08/losparques-y-jardines-son-importantes.html

- McMullan, M.; Donegan, T. M. y Quevedo, A. (2010). Field guide to the birds of Colombia. Bogotá:ProAves.

- Osorio, J. y Molina, L. F. (2009). A vuelo de pájaro. Las ciudades como refugio para las aves. Revista Nodo,4(7), 47-58.

- Remsen, J.V.; Cadena, C.D.; Jaramillo, A.; Nores, M.; Pacheco, J.F.; Robbins, M.B.; Schulenberg,T.S.; Stiles, F.G.; Stotz, D. F. y Zimmer, K. J. (2009).A classification of the bird species of South America. http://www.museum.Isu.edu/ Remsen/SACCBaselin e.html

- Renjifo, L. M., A. M. Franco, H. Álvarez-López, M. Álvarez, R. Borja, J.E. Botero, S. Córdoba, S. De la Zerda, G. Didier, F. Estela, G. Kattan, E. Londoño, C. Márquez, M. I. Montenegro, C. Murcia, J. V. Rodríguez, C. Samper y W.H. Weber. 2000. Estrategia nacional para la conservación de las aves de Colombia. Instituto Alexander von Humboldt, Bogotá, Colombia. ISBN 958-

- Renjifo, L. M.; Franco, A. M.; Amaya, J. D.; Kattan, G. H. y López, B. (2002). Libro rojo de aves de Colombia. Serie Libros Rojos de Especies Amenazadas de Colombia. Bogotá: Instituto de Investigación de Recursos Biológicos Alexander von Humboldt y Ministerio del Medio Ambiente.

- Salaman, P.; Donegan, T. \& Caro, D., (2009). Listado De aves de Colombia 2009. Conservación Colombiana, 8: 1-89 http//:www.proaves.org

- Sociedad Antioqueña de Ornitología -SAO-. (2009). Vida, color y canto. Plantas neotropicales que atraen aves. Medellín: SAO.

- Serrano, V. H. y Aparicio, A. (1996). Biodiversidad de aves a nivel del paisaje en bosques andinos y estudio de sus procesos dinámicos. Cali:Fundación para la Promoción de la Investigación y la Tecnología.

- Villegas M. y Garitano, A. (2008).Las comunidades de aves como indicadores ecológicos para programas de monitoreo ambiental en la ciudad de La Paz, Bolivia. Ecología en Bolivia, 43(2), 146-153. 
1. Administrador del Medio Ambiente. Taxidermista. Universidad Tecnológica de Pereira. juanca112@gmail.com 